\title{
Hybrid versus traditional cardiac rehabilitation models: a systematic review and meta-analysis
}

\author{
Congyin $\mathrm{Wu}^{1}$, Yan $\mathrm{Li}^{2}$, Juan Chen ${ }^{1}$ \\ 'Department of Healthcare Centre, People's Hospital of Hainan Province, Haikou, China \\ 2Department of Emergency Medicine, Orthopaedics, and Diabetes, Hospital of Haikou City, Haikou, China
}

\begin{abstract}
Background: The common drawbacks of standard cardiac rehabilitation (CR) models include low participation rate, high cost, and dependence on on-site exercise sessions. Therefore, hybrid CR protocols have been developed.

Aim: We aimed to test whether hybrid CR models are superior or equivalent to the traditional CR models in patients after myocardial infarction, heart failure, and cardiac surgery, using a meta-analysis framework.

Methods: Data from relevant original studies indexed in the Medline, Scopus, Cochrane Central, and Web of Science databases were extracted and analysed. The standardised mean difference (SMD) was used as a summary effect estimate, along with 95\% confidence interval $(\mathrm{Cl})$.

Results: Based on data from 1195 patients, the summary effect size showed similar improvement in functional capacity in hybrid and standard CR programmes (SMD $=-0.04,95 \% \mathrm{Cl}-0.18$ to $0.09, \mathrm{p}=0.51$ ). No significant difference was detected between the two models in terms of changes in exercise duration ( $\mathrm{SMD}=-0.14,95 \% \mathrm{Cl}-0.51$ to $0.24, \mathrm{p}=0.47$ ), systolic $(\mathrm{SMD}=-0.01,95 \% \mathrm{Cl}-0.14$ to $0.12, \mathrm{p}=0.91)$, and diastolic (SMD $=-0.03,95 \% \mathrm{Cl}-0.16$ to $0.11, \mathrm{p}=0.7)$ blood pressure, or health-related quality of life (SMD $=-0.08,95 \% \mathrm{Cl}-0.23$ to $0.07, \mathrm{p}=0.27)$. In terms of blood lipids, no significant difference was noted between hybrid and traditional CR models in all assessed lipid profile parameters, except for triglycerides (favouring the traditional CR model).

Conclusions: Hybrid CR protocols showed comparable efficacy to the traditional model. Further well-designed studies are required to validate these findings, especially regarding the long-term outcomes.
\end{abstract}

Key words: cardiac rehabilitation, functional capacity, hybrid, meta-analysis

Kardiol Pol 2018; 76, 12: 1717-1724

\section{INTRODUCTION}

Cardiovascular diseases (CVDs) are the most common cause of mortality worldwide (around one-third of all deaths around the world). The World Health Organization estimated an annual global death rate of 17.7 million from all CVDs and 7.4 million from coronary heart disease [1]. Due to advancing health care services, the rate of survival from acute cardiac conditions is increasing; however, patients remain at high risk of recurrence and mortality, especially when they do not adhere to risk factor-modification programmes [2].

Cardiac rehabilitation (CR) is a secondary prevention measure that involves physical exercise, health education, behavioural change, risk factor modification, and nutritional and lifestyle counselling [3,4]. Growing evidence has shown that it can reduce morbidity and mortality and enhance the patients' quality of life [5-8]; therefore, it has become a class I recommendation in several guidelines for patients after cardiac events or surgery and those with heart failure (HF) $[9,10]$. Despite all the known health benefits of CR programmes, the patients' commitment and adherence are still low [11, 12]. This may be attributed to many factors, including low referral rate, lack of motivation, comorbidities, distant CR centres, work or family commitments, and financial or insurance problems $[13,14]$. Due to these limitations of centre-based $\mathrm{CR}$, home-based CR programmes have been considered as an alternative. Home-based CR may be delivered by a therapist at home [9] or monitored by telephone, internet, or videoconference (telerehabilitation) [15].

Due to the possibility of acute cardiac events occurrence during exercise (which need immediate medical attention),

Address for correspondence:

Dr. Congyin Wu, Department of Healthcare Centre, People's Hospital of Hainan Province, 43 Renmin Ave, Meilan Qu, Haikou Shi, Hainan Sheng, China, 570208, tel: +8615289897514,e-mail: wcycx2007@163.com

Received: 13.06.2018 Accepted: 7.08.2018

Available as AoP: 9.08.2018

Kardiologia Polska Copyright (c) Polish Cardiac Society 2018 


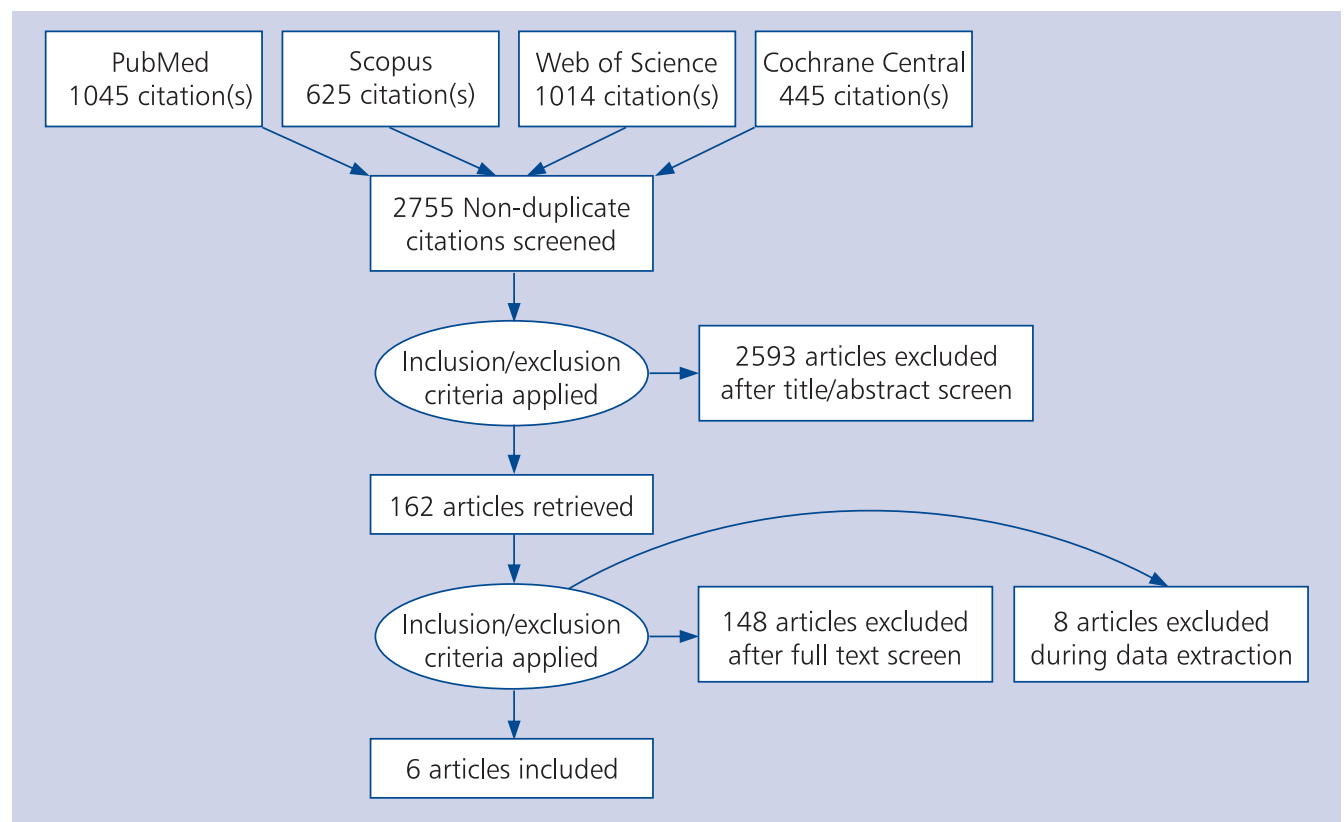

Figure 1. PRISMA flow diagram of search results and study selection

the safety of home-based CR has been questioned [5]. To address the barriers of centre-based CR and the safety aspect of home-based CR, new models have been developed, such as hybrid CR. The hybrid CR model is a combination of home-based CR and direct supervised centre-based CR [16]. We conducted this systematic review to assess the efficacy of the hybrid CR model in comparison to standard CR in patients after cardiac events or interventions and those with HF.

\section{METHODS}

This study was reported in accordance with the preferred reporting items for systematic review and meta-analysis (PRISMA) statement guidelines (Suppl. Table S1 - see journal website) [17].

\section{Literature search strategy}

We conducted a computerised search of the Medline via PubMed, Cochrane register of randomised controlled trials (CENTRAL), Scopus, and Web of Science (WOS) databases, using variations of the following search terms: "cardiac rehabilitation," "cardiovascular rehabilitation," "home-based," "hybrid," "classic," "traditional," "standard," "hospital-based," and "centre-based." We did not apply any search limitations or filters in any of the searched databases. The search was done on $15^{\text {th }}$ April 2018 and again on $15^{\text {th }}$ July 2018. Moreover, a manual search of the reference lists of the included articles was performed for any missing eligible studies.

\section{Inclusion and exclusion criteria}

We included randomised controlled trials (RCTs), non-RCTs, and observational studies that included patients with recent cardiac events or interventions or those with $\mathrm{HF}$, and we compared hybrid CR to standard CR. We considered studies that reported at least one of the following outcomes: health-related quality of life (HRQoL), maximal workload, exercise duration (ED, min), functional capacity measured by metabolic equivalents (METs), systolic (SBP) and diastolic (DBP) blood pressure, lipid profile, or cardiopulmonary exercise testing measures $\left(\mathrm{VO}_{2}, \mathrm{VO}_{2 \text { peak' }}\right.$ or $\mathrm{VO}_{2}$ at aerobic threshold). There was no restriction regarding the date or language of publications.

\section{Study selection and data extraction}

The search results were imported to EndNote X8.0.1 software (Clarivate Analytics, Philadelphia, PA, USA). After duplicate removal, two independent authors performed the title and abstract screening. Articles that met our criteria were retrieved for full-text screening, which was done by two other independent authors. Any disagreements were solved by discussion. The two authors independently extracted the following data from each study: first author, year of publication, study location, sample size, patients' basic characteristics, programme duration, monitoring method, inclusion and exclusion criteria, main results, and main outcome data.

\section{Statistical analysis}

The analysis of the obtained data was done on RevMan 5.3 software (Cochrane Collaboration, London, UK). We used the standardised mean difference (SMD) and the inverse variance method for pooling data of continuous outcomes. The fixed effect model was used. Heterogeneity was assessed by the $\chi^{2}$ test, and its extent was measured by the $\mathrm{I}^{2}$ statistical test. 
According to the Cochrane handbook for systematic reviews of interventions, a significant heterogeneity was considered if p-value of the $\chi^{2}$ test was below 0.1 , and the $I^{2}$ test was interpreted as follows: $0 \%-40 \%$, may not be important; $30 \%-60 \%$, may represent moderate heterogeneity; $50 \%-90 \%$, may represent substantial heterogeneity.

\section{RESULTS}

\section{Search results and baseline characteristics}

Database searching resulted in obtaining 2730 unique citations. After title and abstract screening, 160 articles were found to be eligible for full review. A total of 13 papers were further included for data extraction, of which six articles were included in the final analysis (Fig. 1) [2, 5, 11, 18-20]. Five studies were conducted between 2011 and 2017, and one study was conducted in the United States in 2000. The studies took place in Canada, the United States, Poland, and Iran. Four of the included studies were quasi-experimental, one was a retrospective study, and one was an RCT. The mean age of the enrolled participants ranged between 51 and 68 years. The design, patients' baseline characteristics, and findings of included studies are reported in Table 1.

\section{Meta-analysis outcomes}

Functional capacity

Five studies (1085 patients) reported the change of functional capacity in the participants, as measured by METs. No significant heterogeneity was detected among the included studies $\left(I^{2}=12 \%, p=0.34\right)$; therefore, the fixed effect model was employed. The overall effect estimate showed no significant difference between hybrid and standard CR models in terms of participants' functional capacity (SMD $=-0.04,95 \%$ confidence interval $[\mathrm{Cl}]-0.18$ to $0.09, \mathrm{p}=0.51$; Fig. $2 \mathrm{~A}$ ).

\section{Exercise duration}

Two homogenous studies (115 patients) reported the change in $\mathrm{ED}$ with the two CR models $\left(\mathrm{I}^{2}=64 \%, \mathrm{p}=0.10\right)$. The hybrid and traditional CR models were comparable in terms of the duration of participants' physical exercise $(\mathrm{SMD}=-0.14$, $95 \% \mathrm{Cl}-0.51$ to $0.24, \mathrm{p}=0.47$ ) (Fig. $2 \mathrm{~B}$ )

\section{Physical activity}

Physical activity, measured by the Godin Leisure-Time Exercise Questionnaire, was assessed by one study that showed comparable results between the hybrid and traditional CR models $(\mathrm{SMD}=0.06,95 \% \mathrm{Cl}-0.31$ to $0.44, \mathrm{p}=0.75$ ) (Fig. 2C).

\section{Blood pressure parameters}

The resting SBP and DBP values were reported by five studies that enrolled 1085 participants. No heterogeneity was found among these studies $\left(I^{2}=0 \%, p>0.1\right)$. Under the fixed effect model, no significant difference was detected between the hybrid and standard CR programmes in either
$\mathrm{SBP}(\mathrm{SMD}=-0.01,95 \% \mathrm{Cl}-0.14$ to $0.12, \mathrm{p}=0.91)$ or DBP $(\mathrm{SMD}=-0.03,95 \% \mathrm{Cl}-0.16$ to $0.11, \mathrm{p}=0.7$ ) (Fig. 3A, B).

\section{Quality of life}

Two homogenous studies (890 patients) provided data on the change in HRQoL $\left(I^{2}=27 \%, p=0.24\right)$. The pooled SMD showed no significant difference between the hybrid and traditional CR models (SMD $=-0.08,95 \% \mathrm{Cl}-0.23$ to 0.07 , $p=0.27$ ) (Fig. 4A).

One study by Saeidi et al. [19] assessed the effect of the hybrid and traditional CR models on chest pain intensity and discomfort after cardiac surgery. Both the hybrid and traditional models effectively improved chest pain discomfort and intensity with no significant difference between the two models (SMD $=0.04,95 \% \mathrm{Cl}-0.34$ to $0.42, \mathrm{p}=0.83$ ) (Fig. 4B, C).

\section{Lipid profile}

Three homogenous studies (970 patients) reported the changes in the lipid profile of patients who received traditional and hybrid CR. Participants in the hybrid and traditional CR models had their lipid profiles improved by $10 \%$ to $20 \%$; however, the two models were similar in terms of changes in total cholesterol $(\mathrm{SMD}=0.11,95 \% \mathrm{Cl}-0.03$ to $0.26, \mathrm{p}=0.11)$, low-density lipoprotein cholesterol $(\mathrm{SMD}=0.05,95 \% \mathrm{Cl}$ -0.09 to $0.19, p=0.46)$, and high-density lipoprotein cholesterol levels (SMD $=0.01,95 \% \mathrm{Cl}-0.13$ to $0.15, \mathrm{p}=0.87$ ). A significant difference that favoured the traditional CR model was found only in serum triglyceride levels $(S M D=0.17$, 95\% Cl 0.02 to 0.32, p = 0.03, two studies) (Fig. 5A-D).

\section{DISCUSSION}

Cardiac rehabilitation is a secondary prevention programme that has been shown to prevent event recurrence and improve survival [21]. Low participation in the traditional on-site CR programmes $[22,23]$ has urged health care providers to develop alternative models [24, 25]. Home-based CR programmes, in which the participants can complete their exercises independently at home, have shown equivalent outcomes to the standard CR models [26]. Some patients are, however, ineligible for home-based CR due to the increased risk of acute events occurrence during exercise. Therefore, hybrid models were developed to provide a partially outpatient and partially telemonitored, home-based CR programme [11]. This has been reinforced by the recent successes of telemedicine in several emergency and rehabilitation indications [27-29]. In this study, we aimed to provide class I evidence regarding the comparability of hybrid CR models to the standard CR programmes.

Our analysis showed that hybrid CR models and traditional CR programmes achieved comparable results in terms of improvements in the physical capacity and ED of cardiac patients. This improvement was linked to reduced mortal- 


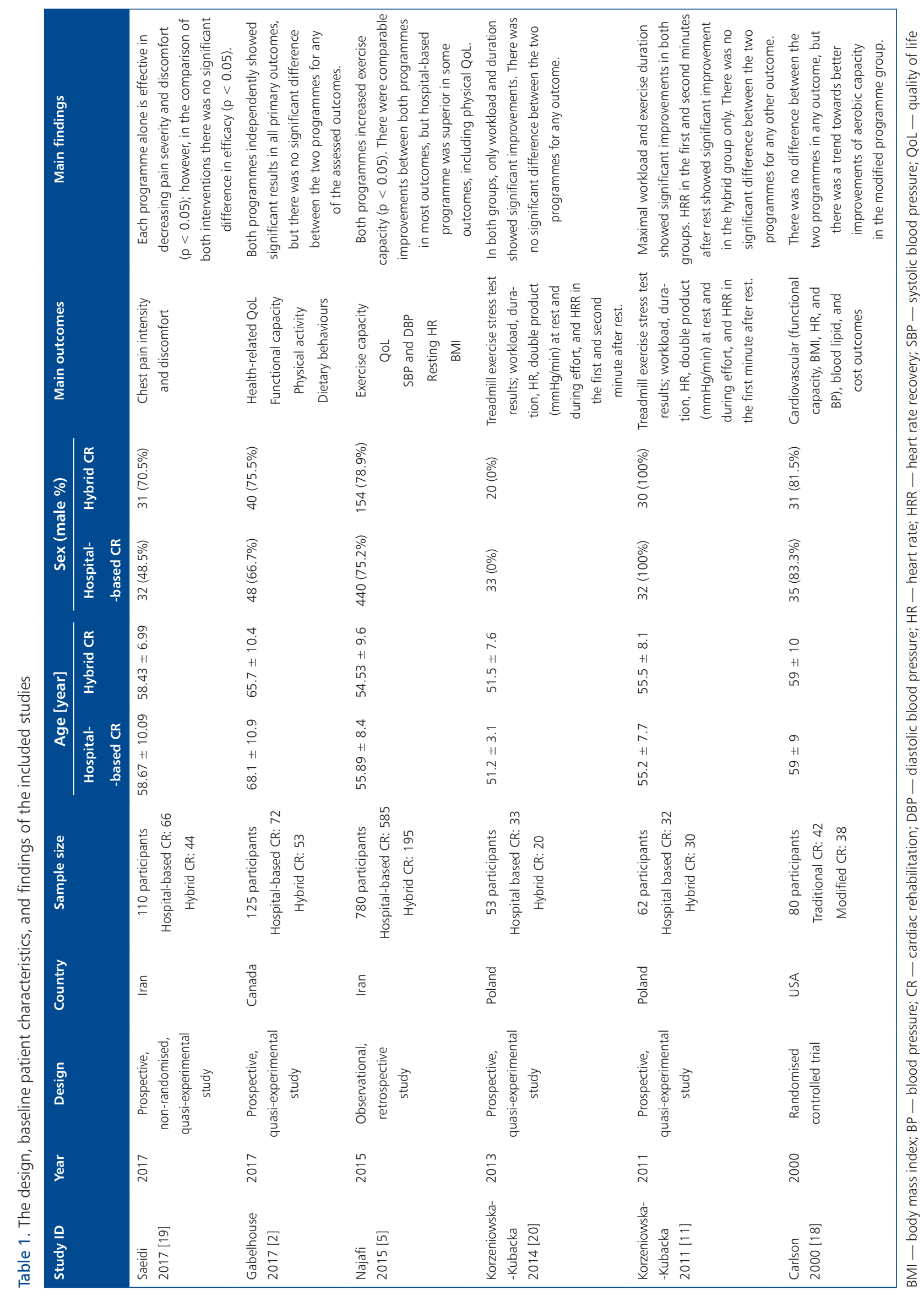




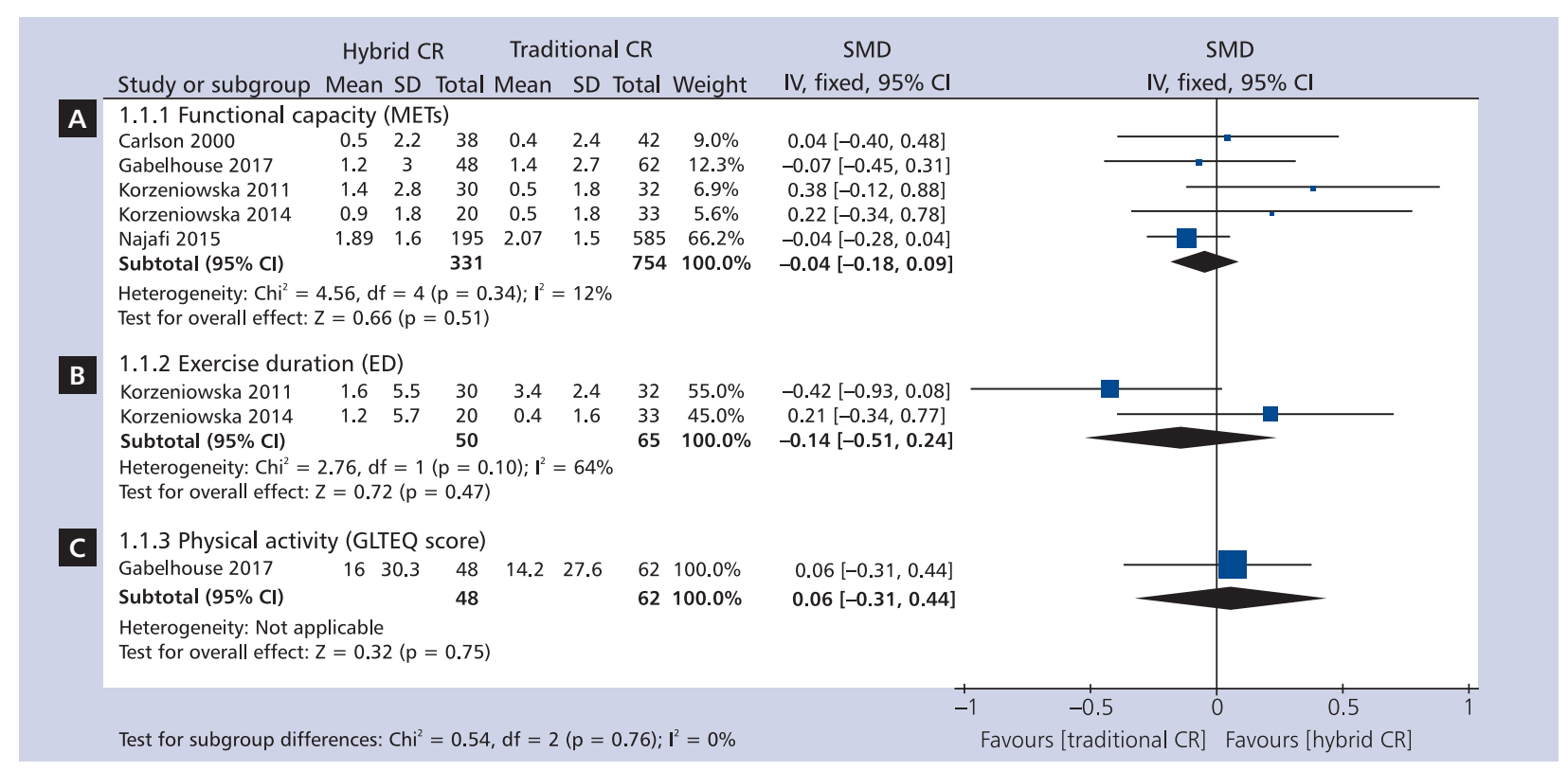

Figure 2. Forest plots of functional capacity (measured by metabolic equivalents [METs]) (A), exercise duration (B), and physical activity (C); $\mathrm{Cl}$ - confidence interval; CR — cardiac rehabilitation; SD — standard deviation; SMD — standardised mean difference

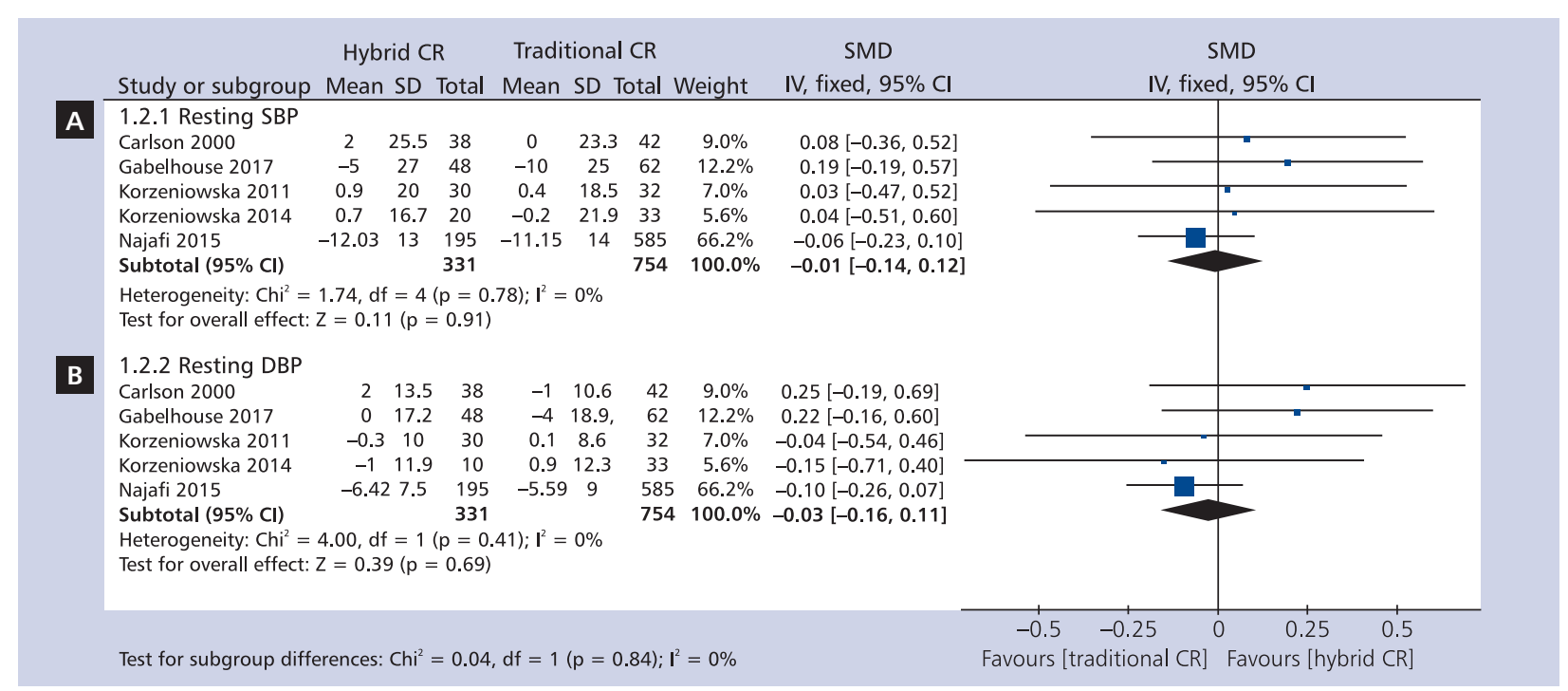

Figure 3. Forest plot of changes in systolic blood pressure (SBP) (A) and diastolic blood pressure (DBP) (B); abbreviations — see Figure 2

ity rates in cardiovascular patients [30, 31]. Furthermore, we found no significant differences in cardiac parameters, including SBP and DBP, between the hybrid and standard models. Improvement of HRQoL throughout CR was also similar between the traditional and hybrid models, with no significant adverse effects of telemonitoring. In addition, changes in the cardiovascular risk factors including the lipid profile of the participants were mostly similar in the two CR approaches.
Heart rate recovery (HRR) is the difference between heart rate at maximal physical effort and in the first or second minute of the recovery period. A heart rate drop by $>12 \mathrm{bpm}$ in the first minute and $>22 \mathrm{bpm}$ in the second minute reflects proper reactivation of the parasympathetic nervous system [11]. Korzeniowska-Kubacka et al. [11] showed that HRR was significantly improved in patients who participated in the hybrid programme but not in those who used the standard CR model. In another study by Giallauria et al. [32] the HRR was 


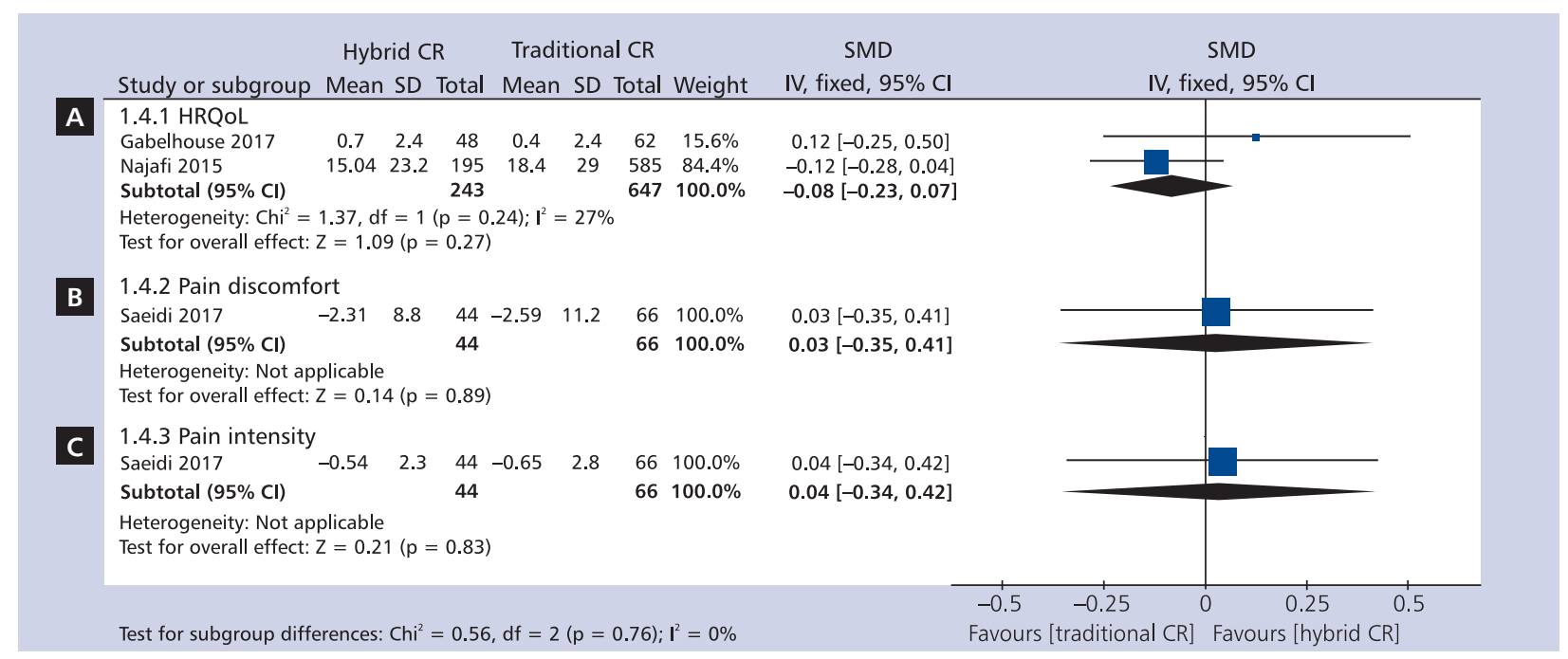

Figure 4. Forest plots of changes in health-related quality of life (HRQoL) (A), pain discomfort (B), and intensity (C); abbreviations - see Figure 2

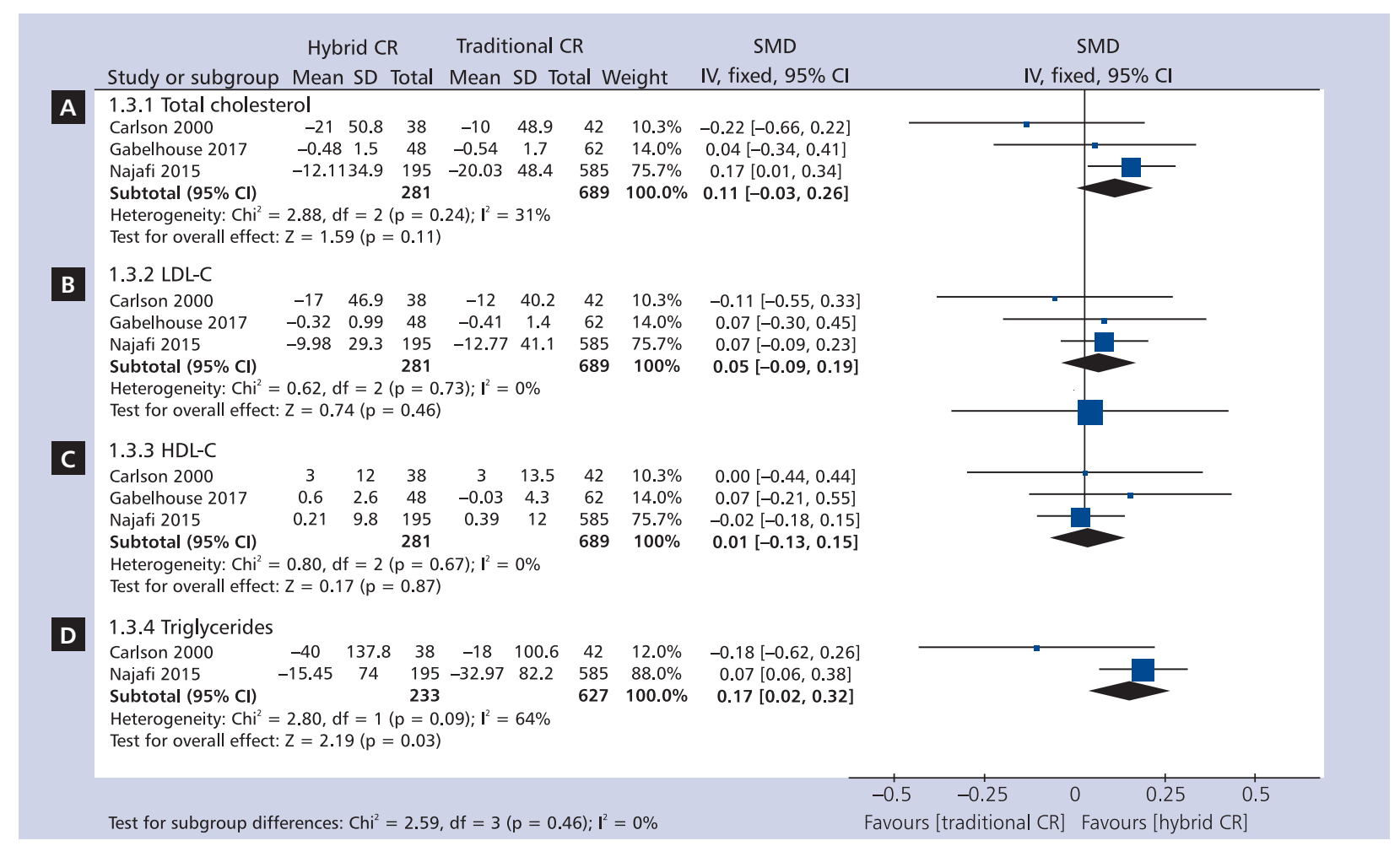

Figure 5. Forest plot of lipid profile changes; A. Total cholesterol; B. Low-density lipoprotein cholesterol (LDL-C); C. High-density lipoprotein cholesterol (HDL-C); D. Triglycerides; abbreviations — see Figure 2

improved by a three-month hospital-based CR programme. The study also demonstrated that this improvement in the parasympathetic reactivation could be maintained by continuation via a hybrid home-based standard programme.
Among the included studies, the participants in the hybrid CR model had either similar or higher adherence (fewer dropouts) as compared to the traditional CR model. Carlson et al. [18] argued that promoting independent exercise and 
higher flexibility of the hybrid programme structure could have contributed to improving the participation. The hybrid model included fewer on-site visits and initiated an "open gym" format for the participants, which promoted fruitful participation. Conversely, supervision during the standard CR programme might have enhanced the dependence on hospital-based exercise and diminished independent exercise. The hybrid model was associated with fewer electrocardiogram sessions, which contributed to a significant cut to the costs of the programme. In addition, lower staff needs in the hybrid CR model resulted in extra savings [18]. This is particularly favourable in low- and moderate-risk patients because high-risk patients may require standard monitoring.

This study has some limitations. First, our analysis included a small number of eligible studies. Second, the analysed studies were predominantly quasi-experimental and observational, with only one RCT included. This is due to the lack of larger trials in this area. Third, due to the lack of data, we could not analyse the differences between both models in terms of cardiopulmonary exercise testing parameters. Well-designed trials with long-term follow-up are imperative to substantiate these findings. Future studies should also investigate the effect of both models on other outcomes, such as $\mathrm{VO}_{2}, \mathrm{VO}_{2 \text { peak' }}$ and $\mathrm{VO}_{2}$ at aerobic threshold, because these outcomes are more objective and are related to functional and exercise capacity [33].

In conclusion, hybrid CR models are associated with equivalent improvements in the physiological and quality-of-life outcomes compared to the standard models. Along with improved participant adherence to the exercise programme, the hybrid models constitute an economically beneficial alternative to standard CR programmes. Larger, well-designed RCTs are warranted to investigate participants' survival and outcomes, especially in the long-term.

\section{Conflict of interest: none declared}

\section{References}

1. World Health Organization. Cardiovascular diseases (CVDs) http://www who int/mediacentre/factsheets/fs317/en/index html (2009).

2. Gabelhouse J, Eves N, Grace SL, et al. Traditional versus hybrid outpatient cardiac rehabilitation: a comparison of patient outcomes. J Cardiopulm Rehabil Prev. 2018; 38(4): 231-238, doi: 10.1097/HCR.0000000000000253, indexed in Pubmed: 29135717.

3. Janssen V, De Gucht V, van Exel H, et al. A self-regulation lifestyle program for post-cardiac rehabilitation patients has long-term effects on exercise adherence. J Behav Med. 2014 37(2): 308-321, doi: 10.1007/s10865-012-9489-y, indexed in Pubmed: 23334387.

4. Balady GJ, Ades PA, Bittner VA, et al. Referral, enrollment, and delivery of cardiac rehabilitation/secondary prevention programs at clinical centers and beyond: a presidential advisory from the American Heart Association. Circulation. 2011; 124(25): 2951-2960, doi: 10.1161/CIR.0b013e31823b21e2, indexed in Pubmed: 22082676.

5. Najafi F, Nalini M. Hospital-based versus hybrid cardiac rehabilitation program in coronary bypass surgery patients in western
Iran: effects on exercise capacity, risk factors, psychological factors, and quality of life. J Cardiopulm Rehabil Prev. 2015; 35(1): 29-36, doi: 10.1097/HCR.0000000000000087, indexed in Pubmed: 25402170.

6. Lawler PR, Filion KB, Eisenberg MJ. Efficacy of exercise-based cardiac rehabilitation post-myocardial infarction: a systematic review and meta-analysis of randomized controlled trials. Am Heart J. 2011; 162(4): 571-584.e2, doi: 10.1016/j.ahj.2011.07.017, indexed in Pubmed: 21982647.

7. Clark AM, Hartling L, Vandermeer B, et al. Meta-analysis: secondary prevention programs for patients with coronary artery disease. Ann Intern Med. 2005; 143(9): 659-672, indexed in Pubmed: 16263889.

8. Taylor RS, Brown A, Ebrahim S, et al. Exercise-based rehabilitation for patients with coronary heart disease: systematic review and meta-analysis of randomized controlled trials. Am J Med. 2004; 116(10): 682-692, doi: 10.1016/j.amjmed.2004.01.009, indexed in Pubmed: 15121495.

9. Frederix I, Hansen D, Coninx K, et al. Telerehab III: a multi-center randomized, controlled trial investigating the long-term effectiveness of a comprehensive cardiac telerehabilitation program-rationale and study design. BMC Cardiovasc Disord. 2015; 15: 29, doi: 10.1186/s12872-015-0021-5, indexed in Pubmed: 25948479.

10. Piepoli MF, Corrà U, Adamopoulos S, et al. Secondary prevention in the clinical management of patients with cardiovascular diseases. Core components, standards and outcome measures for referral and delivery: a policy statement from the cardiac rehabilitation section of the European Association for Cardiovascular Prevention \& Rehabilitation. Endorsed by the Committee for Practice Guidelines of the European Society of Cardiology. Eur J Prev Cardiol. 2014; 21(6): 664-681, doi: 10.1177/2047487312449597, indexed in Pubmed: 22718797.

11. Korzeniowska-Kubacka I, Dobraszkiewicz-Wasilewska B, Bilińska M, et al. Two models of early cardiac rehabilitation in male patients after myocardial infarction with preserved left ventricular function: comparison of standard out-patient versus hybrid training programmes. Kardiol Pol. 2011; 69(3): 220-226, indexed in Pubmed: 21432787.

12. Piotrowicz R, Wolszakiewicz J. Cardiac rehabilitation following myocardial infarction. Cardiol J. 2008; 15(5): 481-487, indexed in Pubmed: 18810728.

13. Beckie TM, Mendonca MA, Fletcher GF, et al. Examining the challenges of recruiting women into a cardiac rehabilitation clinical trial. J Cardiopulm Rehabil Prev. 2009; 29(1): 13-21; quiz 22, doi: 10.1097/HCR.0b013e31819276cb, indexed in Pubmed: 19158582.

14. Moore SM, Dolansky MA, Ruland CM, et al. Predictors of women's exercise maintenance after cardiac rehabilitation. J Cardiopulm Rehabil. 2003; 23(1): 40-49, indexed in Pubmed: 12576911.

15. Hwang R, Bruning J, Morris NR, et al. Home-based telerehabilitation is not inferior to a centre-based program in patients with chronic heart failure: a randomised trial. J Physiother. 2017; 63(2): 101-107, doi: 10.1016/j.jphys.2017.02.017, indexed in Pubmed: 28336297

16. Franklin BA. An alternative approach to the delivery of cardiac rehabilitation services: a "hybrid" model for patient care. J Cardiopulm Rehabil. 2004; 24(6): 383-386, indexed in Pubmed: 15632772 .

17. Moher D, Liberati A, Tetzlaff J, et al. Preferred reporting items for systematic reviews and meta-analyses: the PRISMA Statement. Open Med. 2009; 3(3): e123-e130, indexed in Pubmed: 21603045.

18. Carlson JJ, Johnson JA, Franklin BA, et al. Program participation, exercise adherence, cardiovascular outcomes, and program cost of traditional versus modified cardiac rehabilitation. Am J Cardiol. 2000; 86(1): 17-23, indexed in Pubmed: 10867086.

19. Saeidi M, Soroush A, Komasi S, et al. A hybrid cardiac rehabilitation is as effective as a hospital-based program in reduc- 
ing chest pain intensity and discomfort. Korean J Pain. 2017; 30(4): 265-271, doi: 10.3344/kjp.2017.30.4.265, indexed in Pubmed: 29123621.

20. Korzeniowska-Kubacka I, Bilińska M, Dobraszkiewicz-Wasilewska B, et al. Comparison between hybrid and standard centre-based cardiac rehabilitation in female patients after myocardial infarction: a pilot study. Kardiol Pol. 2014; 72(3): 269-274, doi: 10.5603/KP.a2013.0283, indexed in Pubmed: 24142752.

21. Mendis S, Puska P, Norrving B, et al. Global atlas on cardiovascular disease prevention and control: Geneva: World Health Organization. 2011.

22. Dafoe W, Arthur H, Stokes H, et al. Universal access: but when? Treating the right patient at the right time: access to cardiac rehabilitation. Can J Cardiol. 2006; 22(11): 905-911, indexed in Pubmed: 16971975.

23. Candido E, Richards JA, Oh P, et al. The relationship between need and capacity for multidisciplinary cardiovascular risk-reduction programs in Ontario. Can J Cardiol. 2011; 27(2): 200-207, doi: 10.1016/j.cjca.2011.01.008, indexed in Pubmed: 21459269.

24. Stone JA, Arthur HM. Canadian Association of Cardiac Rehabilitation Guidelines Writing Group. Canadian guidelines for cardiac rehabilitation and cardiovascular disease prevention, second edition, 2004: executive summary. Can J Cardiol. 2005; 21 (Suppl D): 3D-319D, indexed in Pubmed: 16292364.

25. Leung YW, Grewal K, Gravely-Witte S, et al. Quality of life following participation in cardiac rehabilitation programs of longer or shorter than 6 months: does duration matter? Popul Health Manag. 2011; 14(4): 181-188, doi: 10.1089/pop.2010.0048, indexed in Pubmed: 21241185.

26. Dalal HM, Zawada A, Jolly K, et al. Home based versus centre based cardiac rehabilitation: Cochrane systematic review and meta-analysis. BMJ. 2010; 340: b5631, doi: 10.1136/bmj.b5631, indexed in Pubmed: 20085991.

27. Baratloo A, Rahimpour L, Abushouk AI, et al. Effects of telestroke on thrombolysis times and outcomes: a meta-analysis. Prehosp Emerg Care. 2018; 22(4): 472-484, doi: 10.1080/10903127.2017. 1408728, indexed in Pubmed: 29345529.

28. Terkelsen CJ, Nørgaard BL, Lassen JF, et al. Telemedicine used for remote prehospital diagnosing in patients suspected of acute myocardial infarction. J Intern Med. 2002; 252(5): 412-420, indexed in Pubmed: 12528759.

29. Russell TG. Physical rehabilitation using telemedicine. J Telemed and Telecare. 2007; 13(5): 217-220.

30. Jolly K, Taylor RS, Lip GYH, et al. Home-based cardiac rehabilitation compared with centre-based rehabilitation and usual care: a systematic review and meta-analysis. Int J Cardiol. 2006; 111(3): 343-351, doi: 10.1016/j.ijcard.2005.11.002, indexed in Pubmed: 16316695.

31. Pomerleau J, Lock K, Knai C, et al. Interventions designed to increase adult fruit and vegetable intake can be effective: a systematic review of the literature. J Nutr. 2005; 135(10): 2486-2495, doi: 10.1093/jn/135.10.2486, indexed in Pubmed: 16177217.

32. Giallauria F, De Lorenzo A, Pilerci F, et al. Long-term effects of cardiac rehabilitation on end-exercise heart rate recovery after myocardial infarction. Eur J Cardiovasc Prev Rehabil. 2006; 13(4): 544-550, doi: 10.1097/01.hjr.0000216547.07432.fb, indexed in Pubmed: 16874143

33. Balsam P, Główczyńska R, Zaczek R, et al. The effect of cycle ergometer exercise training on improvement of exercise capacity in patients after myocardial infarction. Kardiol Pol. 2013; 71(10): 1059-1064, doi: 10.5603/KP.2013.0261, indexed in Pubmed: 24197587.

Cite this article as: Wu C, Li Y, Chen J. Hybrid versus traditional cardiac rehabilitation models: a systematic review and meta-analysis. Kardiol Pol. 2018; 76(12): 1717-1724, doi: 10.5603/KP.a2018.0175.

\section{WHAT IS NEW?}

Traditional cardiac rehabilitation (CR) programmes have several disadvantages, such as low participation rate, high cost, and dependence on on-site exercise sessions. Therefore, hybrid CR protocols have been developed. In this study, we compared the hybrid and traditional CR models in a meta-analysis framework. Our analysis of 1195 patients showed that the improvements in functional capacity, exercise duration, systolic and diastolic blood pressure, and health-related quality of life were comparable between both models. Moreover, reductions in blood lipid profile parameters were similar, except for triglyceride levels that favoured the traditional CR model. Overall, hybrid CR protocols have comparable efficacy to the traditional model. In addition to their cost-effective merit, hybrid CR models may act as a suitable alternative to traditional CR protocols. 